\title{
Transformation durch Purpose, Digitalisierung und Nachhaltigkeit
}

\author{
Alan Hippe $\mid$ Stephan Paul*
}

Bei der Bekämpfung der Corona-Pandemie steht die Healthcare-Industrie im Fokus. Unabhängig von den aktuellen Herausforderungen muss auch sie sich durch Digitalisierung und Nachhaltigkeit wandeln. Der Chief Financial and Information Officer von Roche, dem weltweit größten Biotech Unternehmen, über den Einfluss des Purpose eines Unternehmens auf Kultur und Führung, Innovation und Motivation, Dezentralität und kreatives Arbeiten. Alan Hippe im Gespräch mit Stephan Paul zur Transformation der Wirtschaft.

Paul: Herr Hippe, als CFO eines weltweit führenden Healthcare-Unternehmens können Sie uns wertvolle Hinweise geben, wie man den wirtschaftlichen Wandel mittels Innovation gestaltet. Lassen Sie uns aber mit der Pandemie beginnen. Als sie ausbrach vor anderthalb Jahren, wie hoch ging da Ihr Puls?

Hippe: Es war ein Schock. Nicht nur für das Finanzsystem des Unternehmens, sondern für jeden einzelnen Mitarbeiter. Eine solche Situation beinhaltet aber immer auch die Chance, Dinge neu zu erfahren. Der Wandel, mit dem wir uns auf die Situation eingestellt haben, ist vielfach gelungen.

Was waren für Ihr Unternehmen die dramatischsten Ereignisse im Lauf der letzten anderthalb Jahre?

Dramatisch war die Sorge um die Mitarbeiter. Heute wissen wir wesentlich mehr über das Virus. Damals sind wir von einem auf den anderen Tag ins Homeoffice gegangen und jeder hat sich gefragt: „Wie groß ist das Risiko eigentlich?" Für uns stand zudem die Frage im Raum: „Was können wir als

\footnotetext{
Dr. Alan Hippe | Chief Financial \& Information Officer | Roche

Prof. Dr. Stephan Paul | Lehrstuhl für Finanzierung und Kreditwirtschaft | Ruhr-

Universität Bochum
} 
Diagnostik- und Pharma-Unternehmen zur Pandemiebekämpfung beitragen?" Im vergangenen Jahr hat Roche in der Diagnostik allein 2,6 Milliarden Umsatz mit Produkten gemacht, von deren Existenz wir Anfang 2020 noch nichts wussten. In sehr kurzer Zeit hat Roche viele Produkte entwickelt, die dazu beigetragen haben, dass wir die Pandemie besser managen können. Das macht mich stolz.

\section{Roche produziert aber keinen Impfstoff gegen Corona?}

Impfstoff ist nicht unsere Domäne. Wir sind stark in der Onkologie, der Multiple Sklerose-Forschung oder auch der Ophthalmologie, also der Augenheilkunde. Ein Unternehmen sollte sich immer auf seine Stärken fokussieren. Genau das haben wir gemacht. Im Kontext der Pandemie waren das zum einen die Testverfahren. Außerdem haben wir uns damit beschäftigt, welche Behandlungen auf medizinischer Seite Sinn machen. Womit können wir jemandem helfen, der an Covid erkrankt? Dazu entwickeln wir Lösungen.

Wann wird es Ihrer Ansicht nach ein leistungsfähiges, für jeden erschwingliches Medikament gegen COVID geben?

Zunächst einmal müssen wir die Gesamtsituation in den Griff bekommen. Unsere Projektionen reichen bis in die nächsten zehn Jahre. Und Behandlungen sind davon abhängig, wie stark man erkrankt. Wir beobachten sehr unterschiedliche Formen, von symptomfrei bis schwer belastet - für alle Phasen der Erkrankung wird es Medikamente geben. Im Moment arbeiten wir an Produkten, die die Symptome mindern und eine Übertragung des Virus verhindern können. Da gibt es, wie immer in der Forschung, auch Fehlschläge und Risiken. Aber wir werden noch in diesem Jahr genügend Daten erhalten und sehen, was funktionieren könnte.

Als global tätiges Unternehmen sind Sie auch in globale Lieferketten eingebunden. Bereits zu Beginn der Pandemie gab es Probleme mit Zulieferungen aus China oder auch aus Indien. Verändert das Ihren Blick? Wollen sie u.U. wieder mehr in Europa bzw. in der Schweiz produzieren?

Wir sind ein sehr großes und sehr globales Unternehmen. Auch wenn sich das pandemiebedingt ändert: Roche war im vergangenen Jahr das größte Pharmaunternehmen in den USA. Unsere Lieferketten sind weltumspannend. Wir haben starke und vollständige Wertschöpfungsketten in den USA, in Europa, aber auch in China. Sie betreffen alles, bis zur Forschung. Und auch wenn es Engpässe gab - es wäre keine gute Idee, alles plötzlich in einem Land behalten zu wollen. 
Auch bei Ihnen hat der Trend zum Homeoffice eingesetzt. Roche ist aber ein Unternehmen, das von Forschung, Entwicklung, Innovation, also hochgradig kreativem Arbeiten lebt. Wie gelingt es, die notwendige Kreativität beim dezentralen Arbeiten zu wahren?

Die Umstellung auf Homeoffice ist uns vergleichsweise leicht gefallen. Innovationen entstehen aber an Schnittstellen und im Austausch, oft auch im nicht geplanten Austausch, z.B. in der Kaffee-Ecke. Und Sie haben Recht: Wir sind davon abhängig, Innovationen zu schaffen. Das ist unser Geschäftsmodell. Roche gibt allein über 12 Milliarden US-Dollar im Jahr für Forschung und Entwicklung aus. Letztlich sind wir angesichts der Pandemie sehr differenziert vorgegangen: Wir haben dafür gesorgt, dass unsere Mitarbeiter unter sicheren Bedingungen arbeiten konnten. Wir haben uns darauf fokussiert, die Menschen im Labor oder aus der Fabrikation zu schützen. Im Moment haben wir im Pharmazweig 86 neue Produkte in der Pipeline. Das ist bei weitem die breiteste Pipeline in der Industrie. Und wenn wir jetzt zurückblicken, sind die Beeinträchtigungen durch die Pandemie hier minimal.

Schweißt eine solche Situation ein Unternehmen zusammen oder erhöht sie die Fliehkräfte?

Roche hat einen sehr starken Purpose - auf Deutsch: Zweck oder Bestimmung. Er lautet: „Doing now what patients need next”. Wer in unserem Unternehmen arbeitet, liebt diesen Purpose. Denn er hat die Chance mitzuhelfen, dass es Patienten besser geht. Wir stellen Medikamente her für schwere Krankheiten wie Krebs oder Multiple Sklerose. Das schweißt zusammen. Außerdem haben wir uns vor rund einem Jahr neue, sehr ehrgeizige Ziele gesetzt. Wir nennen sie die „10 year ambitions“. Wir arbeiten gemeinsam an etwas, für das es sich lohnt, jeden Morgen aufzustehen.

Unternehmenskultur kann zum Problem werden, wenn sie zur Erstarrung führt. Wie führt man in einer solchen Situation? Wie motiviert man sein Team und hält es zusammen, wenn die Unsicherheit steigt?

Roche ist ein sehr dezentrales Unternehmen, Top-Down ist keine große Stärke von uns. Natürlich spiegelt eine Führungskultur das Geschäftsmodell wider und gibt Ziele vor. Wir führen aber kontextual. Wir bauen ein Framework auf, zeigen Werte, die wir erreichen wollen. Das allein genügt, um in die richtige Richtung zu weisen. Eine dezentrale Herangehensweise ist vielleicht nicht die effizienteste. Innovation hat aber viel mit Schnittstellen und Experimentieren zu tun. Wer experimentieren will, kann das nicht immer mit größter Effizienz tun. Wir versuchen auf der Führungsebene die Balance zu finden zwischen Dezentralisierung, Freiraum, Empowerment der Mitarbeiter und auf der anderen Seite die Ressourcen genau dort einzusetzen, wo man den größten Impact für Patienten erzielt. Und das ist uns in der Pandemie hervorragend gelungen. 
Hat sich durch die Pandemie in Ihrer Finanzierungspolitik etwas geändert?

Eigentlich nicht. Für uns waren die Veränderungen durch die Finanzkrise entscheidend. Vor der Finanzkrise kamen die Banken zu den Unternehmen und haben nach deren Solvenz gefragt, heute ist das umgekehrt. Vom Grundsatz her hat sich in der Zusammenarbeit aber nicht viel geändert. Wir waren als Roche schon immer direkt, innovativ und proaktiv. Auch Green Financing spielt bei uns schon immer eine Rolle. Man kann nicht gute Dinge für Patienten tun und sich auf der anderen Seite nicht nachhaltig aufstellen.

In der Kommunikation im Kapitalmarkt ist Roche aber vergleichsweise wenig offensiv hinsichtlich Green Finance.

Wir schneiden schon lange im Dow Jones Sustainability Index hervorragend ab. Nachhaltigkeit steckt in unserer DNA. Die stimmrechtsberechtigten Aktien befinden sich zum großen Teil in der Mehrheit der Familie, die im Prinzip noch die Gründerfamilie ist. Wir bringen daher bei diesem Thema eine große Stabilität mit. Und wir haben gesehen, dass Nachhaltigkeit attraktiv ist für Investoren. Ein zweistelliger Milliardenbetrag geht zurück auf Investoren, die auf Nachhaltigkeit fokussiert sind und denen wir dazu Rede und Antwort stehen.

Auch wenn sich an der Finanzierungspolitik nichts verändert hat, versucht man dennoch, mehr Reserveliquidität vorzuhalten, also einen $\mathrm{Fi}$ nanzierungspuffer, falls die Pandemie sich nochmals verstärken sollte?

Wir haben bei Roche ein sehr ausgeprägtes, sehr bekanntes Cash Management, das immer wieder viele Preise gewinnt. Es befähigt uns, den Cashflow von Tag zu Tag zu verfolgen. Wir arbeiten ja nicht nur mit finanzstarken Privatpatienten zusammen, sondern auch mit vielen Hospitälern. Und es hat gut funktioniert. Wir mussten keinen großen neuen Puffer aufbauen, sondern haben einfach verstärkt darauf geachtet, ob die Entwicklung auf der Cash-Seite unseren Erwartungen entspricht. 2019 haben wir einen operativen Free Cashflow von rund 20 Milliarden Schweizer Franken verzeichnet, im Jahr 2020 sank er auf immer noch rund 14 Milliarden Schweizer Franken. Dafür gab es aber auch operative Gründe, die gut erklärbar waren.

Neben der Nachhaltigkeit ist die Digitalisierung einer der Megatrends, die Transformation antreiben. In der Diagnostik-Sparte haben Sie sich der Digitalisierung verschrieben. Wo setzen diese Innovationen im Digitalisierungsbereich an?

Vorweg, das gilt nicht nur für die Diagnostik-Sparte, sondern für das ganze Unternehmen: Gesundheitsdaten werden in Zukunft eine immense Rolle spielen. In der Diagnostik führen wir über 23 Milliarden Tests pro Jahr durch. Teilt man das durch die Weltbevölkerung, testen wir praktisch jeden Menschen auf der Erde dreimal pro Jahr. Wir gewinnen so 23 Milliarden Datenpunkte, anhand derer genauere Diagnosen gestellt werden können. 
In der Onkologie ist das besonders frappierend. Krebserkrankungen sind stark durch Mutationen in DNA und RNA gekennzeichnet. Je besser man diese Mutationen versteht, desto besser wird die Behandlung, die man den Patienten zukommen lassen kann. Diese Behandlungen sind sehr teuer. Auch deshalb ist es wichtig, möglichst schnell die richtige Therapie zu finden. Digitalisierung bedeutet für uns, wichtige Daten zusammenzubringen, um eine umfassende Diagnose des Patienten sicherzustellen. Am Ende steht die richtige Behandlung. Da sehen wir enormen Verbesserungsbedarf.

Vor dem Hintergrund von Datenschutz: Wie sehen Sie die Diskussion zum Thema elektronische Patientenakte? Ist das Steinzeit im Vergleich zu dem, über das Sie verfügen?

Eines muss man klar sagen: Die Daten gehören dem Patienten. Es ist aber heute technologisch möglich, Daten so zu anonymisieren, dass man sie nicht mehr auf einen Patienten zurückführen kann. Der Schatz, der daraus entsteht, ist enorm. Zu wissen, wie bestimmte Patienten auf bestimmte Medikamente in einem bestimmten Zeitablauf reagiert haben, ist entscheidend. Wir führen heute noch klinische Studien durch, bei denen Patienten ein Standardmedikament oder ein Placebo erhalten und eine andere Gruppe ein innovatives Medikament. In der Onkologie ist das enorm frustrierend, denn jeder Teilnehmer setzt große Hoffnung auf das innovative Medikament. Haben wir Daten zur Verfügung, müssen wir keine Patienten in klinischen Studien mehr frustrieren. Und die sinkenden Kosten werden sich im dann auch auf die Medikamentenpreise auswirken.

Forschung ist zeitintensiv, und es gibt eine hohe Scheiterungsrate. Gibt es auch bei Roche die Tendenz, nicht nur selbst zu forschen, sondern junge Start-ups mit innovativen Ideen zu beobachten oder aufzukaufen? BioNTech z.B. kannte vor zwei Jahren in der breiten Öffentlichkeit ja kaum jemand.

Roche ist stark in der Forschung, am stärksten aber sind wir in der Entwicklung. Wir sind erfahren darin, klinische Studien aufzusetzen und arbeiten sehr gut mit Behörden bei Genehmigungen zusammen. Außerdem verfügen wir über das Know-how, ein Medikament am Markt einzuführen und weltweit zu vermarkten. Deshalb arbeiten Start-ups ab einem gewissen Punkt sehr gerne mit uns zusammen. Roche verfügt zudem über die kritische Masse. Im Gegensatz zum Start-up können wir es uns leisten, zu scheitern. Wesentlich ist, dass wir die Sicherheit geben, der Partner zu sein, der das Medikament zur Markteinführung bringt. Eine solche Zusammenarbeit ist also immer eine Synergie. Ich kann mit Stolz sagen, dass wir mit BioNTech schon lange eine Kooperation haben und eng bei Impfstoffen gegen Krebs zusammenarbeiten.

Sie arbeiten immer stärker mit Big Data. Die Big Techs engagieren sich nun auch im Healthcare-Bereich. Wenn Microsoft z.B. Huance aufkauft, 
ist da zu befürchten, dass die Big Techs ernstzunehmende Wettbewerber im Gesundheitsbereich werden?

Vorab stellt sich die Frage: Wie entstehen Hypothesen in der Biologie? Bringe ich einfach eine große Datenmenge mit Artificial Intelligence in Verbindung und hoffe, dass sich irgendeine Korrelation darstellt? Oder stelle ich eine Hypothese auf und prüfe sie anhand von Daten? Wir arbeiten mit den besten Wissenschaftlern der Welt zusammen. Und immer wieder zeigt sich, dass am Ende das biologische Know-how ausschlaggebend ist. In San Francisco haben wir mit Aviv Regev eine Forscherin für Roche gewinnen können, die schon sehr früh Zellen mithilfe von Computerprogrammen modelliert hat. Ohne tiefes biologisches Know-how funktioniert "Computational Biology" nicht. Wir kooperieren mit den großen TechUnternehmen wie Google und nutzen deren Know-how in Artificial Intelligence. Dafür verstehen wir etwas von der Biologie. Ich sehe hier also eher synergetische Effekte als wirklich harten Wettbewerb.

Nochmals zurück zur Finanzierungspolitik. Sie waren bei unterschiedlichen Unternehmen engagiert. Inwiefern ist die jetzige Finanzierungspolitik anders als in den Häusern, für die Sie zuvor tätig waren?

Bei Roche haben wir mit der Einführung der „10 year ambitions“ auch im Finanzierungsbereich eine Art Neustart gewagt. Wir haben unseren Finance Framework zu 100 Prozent auf den Purpose des Unternehmens ausgerichtet: „Doing now what patients need next”. Wir haben ein patientenzentriertes Finance Framework entwickelt und werden Ökosysteme im Bereich Health Care weiter vorantreiben, dies alles unter Berücksichtigung der Kostenfaktoren.

Sehen Sie sich also als einen der zentralen Transformations- Agenten des Unternehmens?

Absolut. Allerdings muss man das im Zusammenhang sehen. Hätte es den Purpose nicht gegeben, gäbe es die „10 year ambitions" nicht. Wir haben weltweit Kosten im Finanzbereich von rund insgesamt 700 Millionen Schweizer Franken. Diese möchten wir noch reduzieren. Inzwischen verfügen wir über einen digitalisierten Backbone auf der ERP-Seite. Die Shared Service Center sind eingerichtet, mit denen wir die ganze Welt bedienen können. Und die Mitarbeiter verfügen über das notwendige Mindset. Die „10 year ambitions“ waren nun genau der Zeitpunkt, alles zu bündeln und umzusetzen. Geld, das im Unternehmen frei wird, investieren wir in Forschung und Entwicklung. Und das setzt enorme Energien frei, auch im Finanzbereich.

Ist die neue Rolle des CFO der Zukunft, dass er weniger aus dem eher vergangenheitsbezogenen Reporting kommt, sondern stärker in die Al und die mit künstlicher Intelligenz erhobenen Datenpools eintaucht, um daraus Steuerungsimpulse für die gesamte Unternehmung zu geben? 
Ganz genau! Der Finanzbereich hat die Chance, das Unternehmen insgesamt zu begreifen. Was er liefern muss, ist Ressourcenallokation, also zu erkennen, wo genau investiert werden muss. Dazu brauchen wir quantitative Daten aus einem digitalisierten Backbone. Wir sprechen heute von „Democratizing Data“, wie bei Google, auf das wir bei Streitereien am Esstisch zuhause zurückgreifen, Fakten nachschauen und dann weiterdiskutieren können. Die Daten stehen jedem zur Verfügung, und man kann ganz einfach Daten zusammenziehen. All dies bedingt ein umfassendes Konzept, das zu 100 Prozent am Purpose des Unternehmens hängt, einem Open Source Approach, in dem man Kritik von außen einholt, und Investitionen in eine weitreichende Digitalisierung. Bei Roche haben wir uns zu einem „Big Bang“ entschlossen: Früher waren alle Finanzdaten ein Mysterium, streng vertraulich! Wir erneuern nun das komplette ERP im Unternehmen, um Jedem im Unternehmen die Daten zur Verfügung stellen zu können, die er benötigt.

Sie brauchen Mitarbeiter, die mit den Daten umgehen können. Verändert sich das Bild des Mitarbeiters in der Finanzabteilung? Muss er eine andere Ausbildung und anderes Know-how mitbringen?

Das Know-how verändert sich - und zwar in mehrerlei Hinsicht. Da ist zum einen das Tech-Know-how, wie ein Grundverständnis von Kodierungen und Algorithmen. Eine große Rolle spielt aber auch das Know-how dafür, dass man als Netzwerkorganisation zusammenarbeitet und sich produktorientiert ausrichtet. Die Finanzabteilung ist kein steifes System mehr, das hierarchisch orientiert ist und am Ende einen Report erzeugt. Man bewegt sich frei im Unternehmen und kann Daten zusammenziehen, um schnell unternehmerische Entscheidungen zu treffen. Und zwar auf Basis eines stabilen, digitalisierten Backbones.

\section{Wagen Sie auch eine Prognose zur Mitarbeiterzahl?}

Da erleben wir einen Trade off. Zum einen bauen wir intern Shared Service Center auf. Zum anderen haben wir Mitarbeiter, die direkt im Business involviert sind, unternehmerische Entscheidungen mitprägen und fokussiert sind auf Ressourcenallokation. Am Ende werden es vielleicht weniger Mitarbeiter sein. Aber durch die Neuausrichtung und das, was man daraus lernt, ergeben sich für jeden, der dazu beiträgt, enorme Chancen.

Eine Frage, die Studierende gerne stellen, lautet: „Wenn wir aus dem Studium ins Arbeitsleben entlassen werden: Auf welche Eigenschaften kommt es in den Veränderungsprozessen, die vor uns liegen, besonders an?"

Gerade im Veränderungsprozess sollte man danach schauen: Welchen Purpose hat das Unternehmen, um welchen Zweck, um welche Bestimmung geht es? Wenn man ein Unternehmen wirklich umfassend danach ausrichtet, folgt alles andere automatisch: Unternehmergeist, Kollaboration, unternehmensbezogenes Denken oder auch „continuos learning“. Aus diesem Grund habe ich auch unsere Finanzstrategie „open source“ gestellt. Wir lernen von außen genau so viel wie von innen. Vor 
Kurzem habe ich z.B. zwölf Weltklasse-CFOs unseren Finanzbereich ganz offen vorgestellt und mit innen diskutiert. Das war sehr inspirierend.

Karrieretipps sind schwierig. Aber gibt es aus Ihrer Erfahrung etwas, was man auf gar keinen Fall tun sollte, oder empfiehlt sich etwas anderes unbedingt?

Jeder Weg ist anders. Die Kunst liegt darin, den eigenen zu finden. Ich komme aus dem Sport. Und mein Trainer hat einmal gesagt: „Fleiß ist ein sehr universelles Talent. " Das stimmt. Ich habe immer versucht, den Job, den ich gerade machte, super zu machen. Alles andere hat sich dann ergeben. Wenn man offen, neugierig und interessiert ist, macht man auch seine Karriere. Und idealerweise gestaltet man sie am Ende so, dass sie einem zuträglich ist. Ich war in Teilen meiner Karriere definitiv zu ambitioniert. Aber in eine Karriere muss man auch investieren. Ich kann nicht auf der einen Seite über Fleiß reden und dann lange über Work-LifeBalance nachdenken. Trotzdem sollte man sich immer fragen: Was will ich genau, was passt zu mir? Wenn man auf dieser Basis Entscheidungen trifft, dann ist man schon ziemlich gut unterwegs.

Also sollte man zunächst seinen persönlichen Purpose finden und dann versuchen, diesen mit dem des Unternehmens in Deckung zu bringen?

Absolut! Das eine geht ohne das andere nicht. Karriere bedeutet auch, dass man opfert. Das ist ja mit vielen Rollen so, die man in der Gesellschaft oder in einem Unternehmen einnimmt. Rollen haben Vorteile, die werden in aller Regel auch gesehen. Aber sie haben oft auch enorme Nachteile. Dessen muss man sich bewusst sein. Und mit diesem Bewusstsein, muss man die Rolle für sich suchen und finden, die man gerne im Unternehmen, in der Gesellschaft oder auch in der Familie einnehmen möchte.

Abschließend: Wie würden Sie den Satz ergänzen: Transformation ist -

...Purpose-getrieben.

Quelle

Das vollständige Gespräch ist erschienen in der Podcast-Reihe „Dialoge zur Transformation der Wirtschaft. Prof. Dr. Stephan Paul spricht mit Entscheidungsträgern über wirtschaftliche Wandlungsprozesse." am 29.05.2021. 